\title{
The Worm Problem of Leo Moser
}

\author{
Rick Norwood, ${ }^{1}$ George Poole, ${ }^{1}$ and Michael Laidacker ${ }^{2}$ \\ ${ }^{1}$ East Tennessee State University, \\ Johnson City, TN 37614, USA \\ ${ }^{2}$ Lamar University, Beaumont, TX 77710, USA
}

\begin{abstract}
One of Leo Moser's geometry problems is referred to as the Worm Problem [10]: "What is the (convex) region of smallest area which will accommodate (or cover) every planar arc of length 1?" For example, it is easy to show that the circular disk with diameter 1 will cover every planar arc of length 1 . The area of the disk is approximately 0.78539 . Here we show that a solution to the Worm Problem of Moser is a region with area less than $\mathbf{0 . 2 7 5 2 4 .}$
\end{abstract}

\section{Introduction}

Some years ago, Leo Moser produced a published list [10] (see also [5] and [11]) of 50 open questions in geometry entitled "Poorly formulated unsolved problems of combinatorial geometry." Problem number 11 in Moser's list is "What is the (convex) region of smallest area which will accommodate (or cover) every planar arc of length 1?" An alternate way to describe Moser's problem is this: What is the size and shape of the flat surface of minimum area that can be used as a hammer head, which upon strategically striking any given planar worm will "smash" the worm simultaneously from stem to stern?"

In an unpublished paper of Laidacker and Poole [9], using the Blaschke Selection Theorem [8], it is shown that the Worm Problem indeed has a solution. That is, there is a convex region of smallest area which will cover every planar arc of given length 1 . However, the solution is not necessarily unique (see Section 4 below). Furthermore, it has been noted in [4] and [16] that the area of a region of solution must exceed 0.21946 . Consequently, the area of a solution region to the Worm Problem lies between 0.21946 and 0.27524 .

In Section 2 progress toward a solution of Moser's problem leading up to the current result is presented. Section 3 contains a proof that the area required for 
a cover of all planar arcs of length 1 is less than 0.27524 . Finally, some remarks and references to conjectures on "improved solutions" are offered in Section 4.

\section{History of the Worm Problem}

If $S$ is the circular disk with diameter 1 and $\alpha$ is a length- 1 arc, then by displacing $S$ so that its center coincides with the midpoint of $\alpha, S$ must include (cover) all the points of $\alpha$. The area of $S$ is less than 0.78539 . Consequently, a solution to the Worm Problem has area less than 0.78539 .

On the other hand, Schaer [13] has constructed the broadest length-1 arc possible, that is, one whose convex hull has minimum width as large as possible. This minimum width is greater than 0.43893 . Consequently, any solution region must cover the straight arc of length 1 , as well as the "broad" arc of Schaer. That is, any solution region must have area at least $(0.43893) / 2 \geq 0.21946$. Hence, the area of any solution region to the Worm Problem lies between 0.21946 and 0.78539 .

Aram Meir showed that a semidisk of diameter 1 will cover any length-1 arc. His simple but very elegant proof is contained in Wetzel's paper of 1973 [17]. The area of this cover is less than 0.39270 , a significant improvement over the disk.

Schaer and Wetzel [14]-[16] have approached the Worm Problem by considering regions whose boundaries are well-known geometric figures such as squares, equilateral triangles, etc. For each class of regions, they determine the dimensions of the region with smallest area which covers all length-1 arcs. In the case of squares, the one with diagonal length 1 is best, and for equilateral triangles, the one with side length slightly greater than 1 is best (see [1] to find why the side length must be larger than anticipated). In either case, the areas of these figures exceed the area of Meir's semidisk.

Wetzel, who has done much to popularize the Worm Problem, used the approach described above in considering the class of sectors $S(r, 2 \Theta)$ where $r$ and $2 \Theta$ denote the radius and central angle, respectively. In [17] Wetzel showed that if $r \geq(0.5) \csc (\Theta)$, then the sector $S(r, 2 \Theta)$ will cover all length-1 arcs. Furthermore, when $r=(0.5) \csc (\Theta)$ and the area of the sector $S(r, 2 \Theta)$ is minimized as a function of $\Theta$, then the resulting sector covers all length-1 arcs and has area less than 0.34510 , an improvement over Meir's semidisk.

In 1972 Gerriets showed that a region with area less than 0.32140 covered all length-1 arcs [3]. Gerriets' region is the union of two regions, one whose boundary is an isosceles triangle with altitude $\frac{1}{4}$ and base length 1 , and one whose boundary is a semi-ellipse with major axis length 1 and minor axis length $\frac{1}{2}$.

Following Gerriets' unpublished solution region, Gerriets and Poole [4] discovered a simple solution region with area less than 0.28870 . The proof is equally simple. Their region is a rhombus with major diagonal 1 and minor diagonal $1 / \sqrt{3}$. Furthermore, by "snipping off' one corner of the rhombus at the end of the minor diagenal, the resulting region has area less than 0.28610 and remains a solution region. To the best of our knowledge, this solution region has not been improved upon for 16 years, that is, until now. 


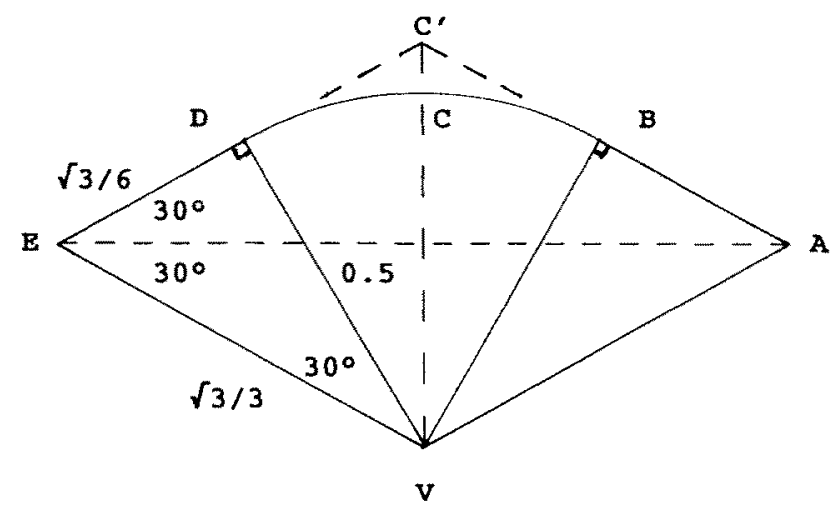

Fig. 3.1

\section{A Smaller Solution Region to Moser's Worm Problem}

The following region is a modification of the rhombus solution of Gerriets and Poole [4]. The region consists of a $60^{\circ}$ sector of radius 0.5 (sector $D V B$ ) with a $30^{\circ} 60^{\circ} 90^{\circ}$ triangle joined to either side, as shown in Fig. 3.1.

Theorem 3.1. Every planar arc of length 1 can be covered by the region represented in Fig. 3.1, whose area is less than 0.27524 .

Preliminary to the proof of Theorem 3.1 we adopt some notation and prove two lemmas. Let $D(X, Y)$ represent the distance from point $X$ to point $Y$ in the plane. Let $\alpha$ denote an arc of length 1 in the plane, the "worm." Any rotation or translation of this arc $\alpha$ is also called $\alpha$. Let $\Theta$ denote the center or midpoint of $\alpha$. Let $b$ be some point on $\alpha$ at least as far from $\Theta$ as any other point on $\alpha$. Call the half-arc of $\alpha$ containing $b$ the $\beta$-arc, or simply $\beta$. Let $t$ be some point on $\alpha$ which is not on $\beta$, and which is at least as far from $\Theta$ as any point not on $\beta$. Call this half-arc of $\alpha$ containing $t$ the $\tau$-arc, or simply $\tau$.

By an arrow we mean any figure composed of three rays emanating from a point $V$, the two outside rays making $60^{\circ}$ angles with the center ray. Throughout this section we always assume that any arrow under discussion is placed in the plane so that

(1) $\alpha$ lies inside the two outside rays of the arrow,

(2) the center ray passes through $\Theta$, the center of $\alpha$, and

(3) $\alpha$ is contiguous with at least one of the two outside rays of the arrow (Fig. 3.2).

For a given $\alpha$, such an arrow is completely determined by the direction it points; for example, a 6 o'clock arrow points down (Fig. 3.2). Of course, the arrows of interest will be those whose rays coincide with segments $V E, V C$, and $V A$ of Fig. 3.1.

For a specific $\alpha$ and given arrow, we then call a line perpendicular to the center 


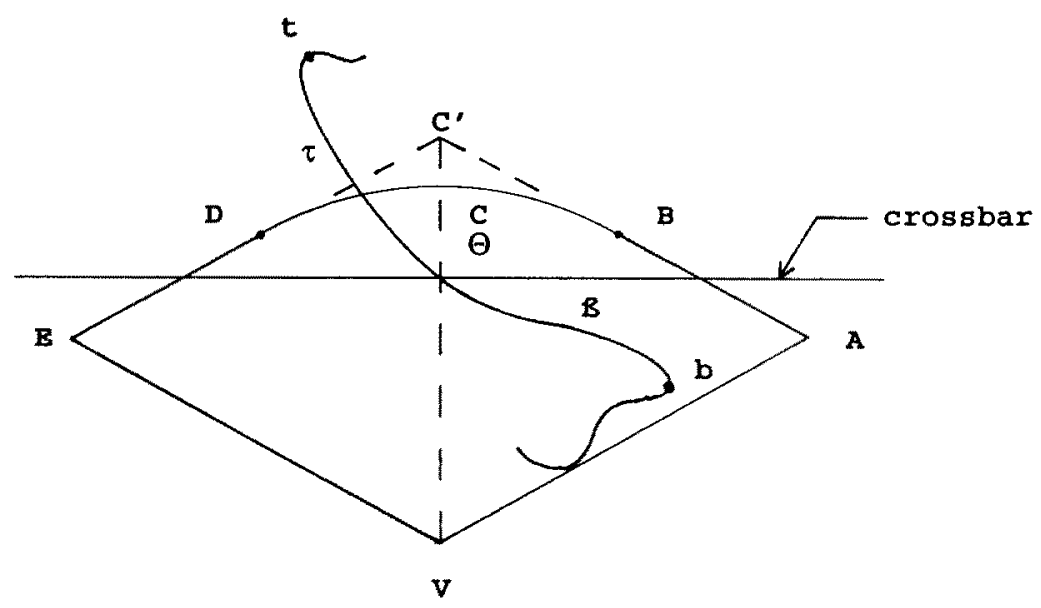

Fig. 3.2

ray at $\Theta$ a crossbar (Fig. 3.2). Furthermore, any point which lies on the same side (opposite side) of the crossbar as $V$ is said to be below (above) the crossbar.

By a rhombus on an arrow we mean the $30^{\circ}-60^{\circ}$ rhombus with major axis 1 which coincides with the arrow at $V$ and along the two outside rays (Fig. 3.2). By the trap on an arrow we mean a region congruent to Fig. 3.1 whose angle $E V A$ coincides with the outside rays of the arrow (Fig. 3.2).

Lemma 3.2 If one side of an arrow touches one half of the worm (either $\beta$ or $\tau$ ), and if $D(\Theta, V) \leq(2 \sqrt{3}) / 9$, then that half-worm is covered by the trap on that arrow (Fig. 3.2).

Proof. Suppose $X$ is one point at which a side of the arrow touches one of the worm halves, say $V A$ touches $\beta$ at $X$. If $\Theta=V$, then $\beta$ (as well as $\tau$ ) lies inside the circle centered at $V$ with radius 0.5 and the entire worm is covered by the trap. Now assume $\Theta$ lies above $V$. There are several cases to consider, and only one of them requires the hypothesis about $D(\Theta, V)$ : the case where the half-worm goes from $\Theta$ through the arc at the top of the trap and then comes back inside the trap to $X$. The other cases are easy. We consider three cases and leave the remaining ones for the reader.

Suppose the half-worm $\beta$ goes from $\Theta$ to $X$ and then crosses segment $A B$ at point $T$. Reflect segment $A B$ in line $V A$ and let $T^{\prime}$ be the reflection of $T$ (Fig. 3.3). If the worm could go from $\Theta$ to $X$ and then cross $A B$ at $T$, then it could go from $\Theta$ to $X$ and then cross $A T^{\prime}$ at $T^{\prime}$. However, the distance from $\Theta$ to $A T^{\prime}$, is $\frac{1}{2}$, so this case is impossible.

Next suppose the half-worm $\beta$ goes from $\Theta$ to $X$ and then across the arc $B D$. For any point $Z$ on $B D$ we have $D(X, Z) \geq D(X, B)$, so this case is impossible.

The other cases are omitted except for the case where $\beta$ goes from $\Theta$ through $Z$ on arc $B D$, and then back inside the trap to $X$. For this last case assume $D(\Theta, V) \leq(2 \sqrt{3}) / 9$. Then the length of the half-worm that escapes must be greater 


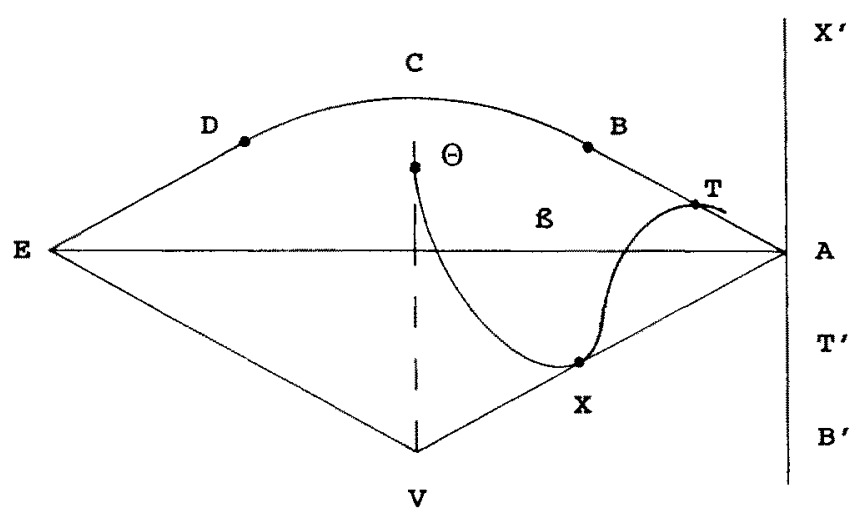

Fig. 3.3

than $D(\Theta, Z)+D(Z, X)$. It can be shown analytically that when $D(\Theta, V) \leq(2 \sqrt{3}) / 9$,

$$
D(\Theta, Z)+D(Z, X) \geq D(\Theta, B)+D(B, X) \geq 0.5
$$

Therefore, this escape is impossible and the half-worm $\beta$ lies inside the trap.

Lemma 3.3. If one side of an arrow touches one half of the worm (either $\beta$ or $\tau)$, and if a point on that half-worm, as distant from $\Theta$ as any point on that half-worm, lies on or above the crossbar for that arrow, then that half-worm is covered by the trap on that arrow (Fig. 3.4).

Proof. Referring to Fig. 3.4, note that each half of the rhombus $E V A C^{\prime}$ is an equilateral triangle. Furthermore, in each equilateral triangle we can inscribe a smaller equilateral triangle measuring $\frac{1}{3}$ on a side, each side being perpendicular to one side of the triangle in which it is inscribed. That is, $D(S, R)=D(S, T)=$

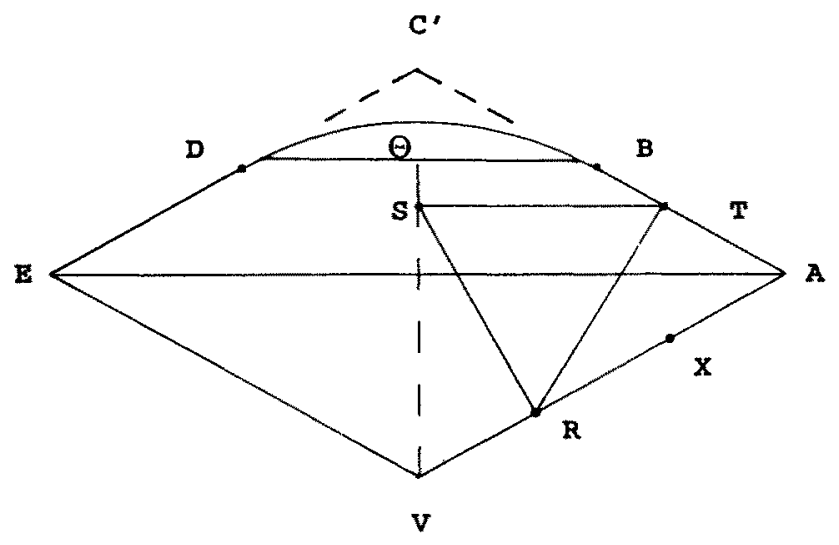

Fig. 3.4 
$\left.D(R, T)=\frac{1}{3}, D(V, S)=(2 \sqrt{3}) / 9\right)$. We will show that under the hypothesis of the lemma, the distance from $V$ to $\Theta$ is less than or equal to $(2 \sqrt{3}) / 9$, hence, Lemma 3.2 applies.

Suppose $\beta$ is the half-worm which satisfies the hypothesis of this lemma, and that it is contiguous with the arrow (say at $X$ on $V A$ ). By way of contradiction, suppose $\Theta$ lies on $S C^{\prime}$ above $S$, as shown in Fig. 3.4. From the work of Gerriets and Poole [4] we know that $\beta$ lies inside the rhombus. Every point inside the rhombus and above the crossbar is less than a distance of $\frac{1}{3}$ from $\Theta$, while for the point $X$ on $\beta, D(\Theta, X) \geq D(S, R)=\frac{1}{3}$. Therefore the point on $\beta$ most distant from $\Theta$ does not lie above the crossbar, contrary to the hypothesis. Consequently, $\Theta$ must lie on the segment $S V$. Since $D(S, V)=(2 \sqrt{3}) / 9$, Lemma 3.2 applies and $\beta$ is covered by the trap.

Proof of Theorem 3.1. If both $\beta$ and $\tau$ simultaneously touch the sides of any arrow while $D(V, \Theta) \leq(2 \sqrt{3}) / 9$, then, by Lemma $3.2, \alpha$ is covered by the trap.

Up to this point, we have tacitly assumed that the arrow points to 6 o'clock. In the remainder of the proof three different types of rotations are considered:

(1) The arc $\alpha$ is rotated keeping the arrow fixed. During this rotation, the center of $\alpha$, denoted by $\Theta$, may move along ray $V C$, so that $\alpha$ lies inside the arrow and is in contact with the arrow at all times.

(2) The arrow is rotated keeping the arc $\alpha$ fixed. During this rotation, ray $V C$ moves back and forth through $\Theta$, to keep the arrow just touching $\alpha$.

(3) Both the arc $\alpha$ and the arrow are simultaneously rotated. During this rotation the distance $D(V, \Theta)$ is fixed.

In each case, the three conditions describing the relationship between $\alpha$ and the arrow prevail. In the first two types of rotations, note that $D(V, \Theta)$ changes during the rotations.

First, we dispose of the special case where one half of $\alpha$ never touches either side of any arrow, as the arrow rotates $360^{\circ}$ around $\alpha$ from 6 o'clock back to 6 o'clock again. Since $b$ is one of the points on $\alpha$ most distant from $\Theta$, it is easy to show that $\beta$ must touch one side of some arrow. In fact, if $\alpha$ is first positioned so that $\Theta b$ is horizontal and $b$ lies to the right of $\Theta$, then $b$ lies on the upper side of the arrow pointing to 4 o'clock. Consequently, if only one half of $\alpha$ is contiguous with any arrow, it must be $\beta$. Thus, we may assume that in this special case $\tau$ never touches any side of any arrow, regardless of the direction in which the arrow points.

By first rotating $\alpha$, position $\beta$ so that $b$ lies directly above $\Theta$. Then for the 6 o'clock arrow, $b$ lies on $V C^{\prime}$. We know that $\beta$ touches one side of the 6 o'clock arrow and that no portion of $\alpha$ lies below the arrow. Since the point $b$ is above the crossbar, by Lemma 3.3 we may infer that $\beta$ is contained in the trap. Also, since $b$ is above $\Theta$, the circle centered at $\Theta$ with radius $D(\Theta, b)$ lies below the top of the trap. Hence, all of $\alpha$ lies below the top of the trap, since $b$ is as far or farther from $\Theta$ than any point on $\alpha$, and $\alpha$ is covered by the trap.

Now that we have disposed of this special case, for the remainder of the proof 


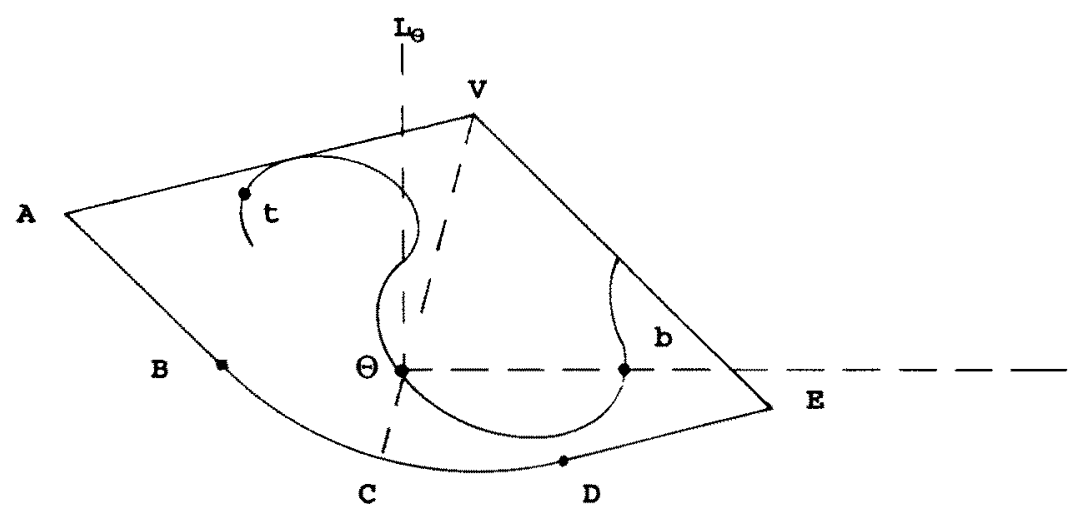

Fig. 3.5

we may assume there is at least one arrow in which $\tau$ is contiguous with one side of that arrow. As noted above, there is always some arrow in which $\beta$ is contiguous with one side. By continuity, if one half of $\alpha$ touches one side of one arrow while the other half of $\alpha$ touches one side of another arrow, then there is some intermediate arrow where both halves, $\beta$ and $\tau$, touch the sides. For the remainder of the proof, we assume there is at least one position of the arrow in which both $\beta$ and $\tau$ are simultaneously contiguous with the arrow. Furthermore, if $\beta$ and $\tau$ are both contiguous and $D(L, \Theta) \leq(2 \sqrt{3}) / 9$, then by Lemma $3.2, \alpha$ is covered by the trap.

Therefore, in the final cases, we make use of Lemmas 3.2 and 3.3 to show that in each case the worm must lie inside the trap.

Given an orientation of the arc $\alpha$ and an arrow which touches both $\beta$ and $\tau$, rotate both $\alpha$ and the arrow together until $\Theta b$ is horizontal and $t$ is on or above the line through $\Theta b$ (Fig. 3.5). Without loss of generality, assume $b$ lies to the right of $\Theta$. There are two cases to consider, which depend on the location of $t$ with respect to a vertical line, $L_{\Theta}$, perpendicular to $\Theta b$ at $\Theta$.

Case 1. Assume $t$ lies on or to the left of $L_{\Theta}$. If $D(\Theta, t) \leq(2 \sqrt{3}) / 9$, we are done. If not, then both $b$ and $t$ lie outside a circle centered at $\Theta$ with radius $\frac{1}{3}$. Since $b$ lies to the right of $\Theta$, any part of $\beta$ which lies to the left of $L_{\theta}$ also lies inside this circle because it lies within 0.5 of $b$, and every point that is outside the circle and to the left of $L_{\theta}$ is more than $(1+\sqrt{2}) / 3$ from $b$. We can therefore position (or construct) two arrows with sides tangent to the circle centered at $\Theta$ with radius $D(\Theta, t)$, such that $t$ is a point of tangency for one of the sides. Since $t$ and $b$ are on opposite sides of $L_{\boldsymbol{\theta}}$, one such arrow points to some time between 9 o'clock and 11 o'clock. From a previous remark we know that if this arrow were rotated counterclockwise around $\alpha$ to 4 o'clock, $b$ would touch. Therefore, there is some arrow between 11 o'clock and 4 o'clock which is contiguous with both $\beta$ and $\tau$. For any such arrow, either $b$ or $t$ lies above the corresponding crossbar (that is to say, on the opposite side of the crossbar from $V$, the vertex of the arrow). As 
shown in the proof of Lemma 3.3, this implies $D(V, \Theta) \leq(2 \sqrt{3}) / 9$. Application of Lemmas 3.2 and 3.3 show that $\alpha$ is covered by the trap.

Case 2. Assume $t$ lies to the right of $L_{\ominus}$ (on the same side of $L_{\circledast}$ as $b$ ). From our assumptions regarding arrows, the 6 o'clock arrow touches either $\beta$ or $\tau$.

Case $2 a$. Assume the 6 o'clock arrow touches $\beta$. Since $b$ is on the crossbar, by Lemma $3.3 \beta$ is covered by the trap. If $\tau$ is also covered by the trap corresponding to the 6 o'clock arrow, we are done. If not, then some point on $\tau$ crosses the top of this trap, and so there is some arrow pointing between 10 o'clock and 12 o'clock which touches $\tau$. Therefore, there is some clockwise rotation of the arrow from 6 o'clock to 12 o'clock which is contiguous with both $\beta$ and $\tau$. For any such arrow, $b$ is above the crossbar. Hence, for this arrow, $D(V, \Theta) \leq(2 \sqrt{3}) / 9$ and $\alpha$ is covered by the corresponding trap.

Case $2 b$. Assume the 6 o'clock arrow touches $\tau$. Then we can rotate the arrow clockwise and at some time before 12 o'clock, one of two things will happen: either both $\beta$ and $\tau$ will touch the arrow in which $b$ lies above the crossbar, or else we reach a point where $\tau$ touches the arrow and in which $t$ lies on the crossbar. In the first situation, Lemmas 3.2 and 3.3 apply and $\alpha$ is covered by the trap. In the second situation we may rotate both $\alpha$ and the arrow together until the arrow points to 6 o'clock. With the roles of $(\beta, b)$ and $(\tau, t)$ reversed, the conditions of Case $2 a$ are satisfied, except for the trivial modification that $\tau$ lies to the left of $\Theta$. This completes the proof of Theorem 3.1.

\section{Remarks and Conjectures on Solution Regions}

While we seek to solve covering problems in the plane, such as the Worm Problem of Moser or any others, there are three important observations which should be made.

First, a solution to a covering problem may not be unique. For example, suppose $L$ denotes the line segment of length 1 while $S$ denotes the region bounded by the square of maximum area which can be inscribed in a semidisk $D$ of diameter 1. Let $\Sigma=\{L, S\}$. In Fig. 4.1, $S_{1}$ and $S_{2}$ are congruent copies of $S$ while $L_{1}=A^{\prime} B^{\prime}$, $L_{2}=A B, L_{3}=D^{\prime} E^{\prime}$, and $L_{4}=D E$ each represent congruent copies of $L . l_{1}$ and

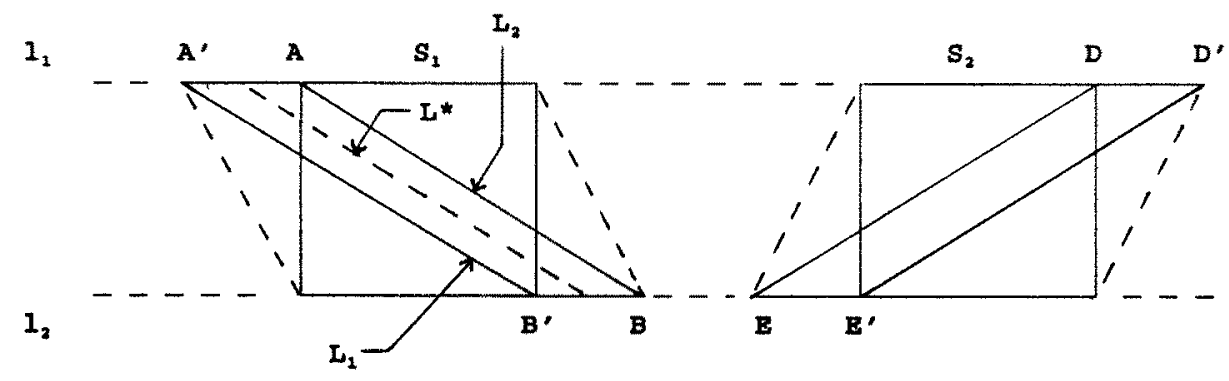

Fig. 4.1 
$l_{2}$ are parallel lines coincident with opposite sides of $S_{1}$ and $S_{2}$ as indicated. Then for each possible line segment $L^{*}$ congruent to $L$ and lying between $L_{1}$ and $L_{2}$ (or $L_{3}$ and $L_{4}$ ), and contiguous at its endpoints with $l_{1}$ and $l_{2}$, consider the convex hull, $C^{*}$ determined by $L^{*}$ and $S_{1}$ (or $L^{*}$ and $S_{2}$ ). Let $\Phi$ denote all such regions $C^{*}$. It can be shown that each element of $\Phi$ is a minimal area cover for the elements of $\Sigma$, and that they are not all equivalent under translation and rotation. Consequently, there are infinitely many distinct minimal covers for the set of elements in $\Sigma$.

Second, the boundary of any minimal solution region need not intersect any of the elements to be covered. For example, if $\Sigma$ denotes the set of all closed disks of radius $r<1$, then the closed disk $C$ of radius 1 is the unique minimal cover for the elements of $\Sigma$. And $C$ can cover any element $B$ of $\Sigma$ without its boundary intersecting any part of $B$.

Finally, perhaps the most important observation is that a straightforward induction type argument will not yield a solution region to a covering problem. For example, if $\Sigma$ is a collection of compact convex sets and $\Sigma^{\prime}=\Sigma-\{\sigma\}$ represents the collection of all but one of the sets in $\Sigma$, then a minimum cover $C$ of $\Sigma$ may not even cover any minimum cover $C^{\prime}$ of $\Sigma^{\prime}$. Specifically, suppose $\Sigma=\{D, S, L\}$ where $D, S$, and $L$ are defined as in the first example of this section. If $\Sigma^{\prime}=\{S, L\}$, then the unique minimum cover of $\Sigma$ is clearly the set $C=D$. However, as noted in the first example, there is a whole family $\Phi$ of sets, each member of which is a minimum cover of $\Sigma^{\prime}=\{S, L\}$. In no case can any member of $\Phi$ be covered by $C$, the minimum cover of $\Sigma=\{D, S, L\}$.

In seeking candidates for solution regions to Moser's Worm Problem with area less than 0.27524 , we should keep in mind four special length-1 arcs: the straight line segment, the block U-shaped arc whose convex hull is a square with side length $\frac{1}{3}$, the "broad worm" of Schaer [13], and the two-angle Z-arc of Besicovitch [1]. Candidate regions must cover these.

Conjecture 4.1 [16]. The sector $S(1, \pi / 6)$ with area less than 0.26180 is a solution region.

Conjecture 4.2 [4]. The $30^{\circ}-60^{\circ}-90^{\circ}$ right triangle, whose smallest side has length $(\sqrt{3}+4) / 6 \sqrt{3}$, is a solution region. Its area is less than 0.26350 .

Conjecture 4.3 [4]. The right isosceles triangle, whose smallest sides have length equal to 1.5 , is a solution region. This region has area 0.25 .

Conjecture 4.4 [4]. The $30^{\circ}-60^{\circ}-90^{\circ}$ right triangle with hypotenuse equal in length to $(6+2 \sqrt{3}) / 9$ is a solution region. Its area is less than 0.23450 .

\section{References}

1. A. S. Besicovitch. On arcs that cannot be covered by an open equilateral triangle of side 1. Math. Gaz. 49 (1965), 286-288. 
2. G. G. Chakerian and M. S. Klamkin. Minimal covers for closed curves. Math. Mag. 46 (1973), 55-61.

3. J. Gerriets. An improved solution to Moser's Worm Problem. Unpublished. 1972.

4. J. Gerriets and G. Poole. Convex regions which cover arcs of constant length. MAA Monthly 81 (1974), 36-41.

5. R. K. Guy. Problems in the Geometry of Metric and Linear Spaces. Lecture Notes in Mathematics, Vol. 490. Springer-Verlag, Berlin. 1975.

6. R. H. Hudson and T. L. Markham. Alfred Brauer. Linear Algebra Appl. 59 (1974), 1-17.

7. P. Jones and J. Schaer. The worm problem. Research Paper No. 100. University of Calgary. 1970.

8. P. Kelly and M. Weiss. Geometry and Convexity. Wiley, New York. 1979.

9. M. Laidacker and G. Poole. On the existence of minimal covers for families of closed bounded convex sets. Unpublished. 1986.

10. L. Moser. Poorly formulated unsolved problems of combinatorial geometry. Mimeographed list. 1966.

11. W. Moser and J. Pach. 100 Research problems in discrete geometry. Mimeographed. McGill University, Montreal. 1986.

12. G. Poole and J. Gerriets. Minimum covers for arcs of constant length. Bull. Amer. Math. Soc. $79(1973), 462-463$.

13. J. Schaer. The broadest curve of length 1. Research Paper No. 52. University of Calgary. 1968.

14. J. Schaer and J. Wetzel. Boxes for curves of constant length. Israel J. Math. 12 (1972), 256-265.

15. J. Wetzel. Triangular covers for closed curves of constant length. Elem. Math. 25 (1970), 78-81.

16. J. Wetzel. On Moser's problem of accommodating closed curves in triangles. Elem. Math. 27 (1972), 35-36.

17. J. Wetzel. Sectorial covers for curves of constant length. Canad. Math. Bull. 16 (1973), 367-376.

Received December 11, 1989, and in revised form June 4, 1990, and July 18, 1991. 